\title{
Serial Number Production Identifier
}

National Cancer Institute

\section{Source}

National Cancer Institute. Serial Number Production Identifier. NCI Thesaurus. Code C101671.

The production identifier is the serial number. 